\title{
Effect of Anticitrullinated Protein Antibody Status on Response to Abatacept or Antitumor Necrosis Factor- $\alpha$ Therapy in Patients with Rheumatoid Arthritis: A US National Observational Study
}

\author{
Leslie R. Harrold, Heather J. Litman, Sean E. Connolly, Sheila Kelly, Winnie Hua, Evo Alemao, \\ Lisa Rosenblatt, Sabrina Rebello, and Joel M. Kremer
}

\begin{abstract}
Objective. Assess whether baseline anticyclic citrullinated peptide antibodies (anti-CCP) status is associated with treatment response in patients with rheumatoid arthritis (RA) initiating abatacept (ABA) or a tumor necrosis factor- $\alpha$ inhibitor (TNFi).

Methods. Using the Corrona RA registry, patients were identified who initiated ABA or a TNFi (June 2004-January 2015), had a followup visit 6 months ( \pm 3 mos) after initiation, and anti-CCP measured at or prior to initiation. Primary outcome was mean change in Clinical Disease Activity Index (CDAI) from initiation to 6 months. Treatment response was evaluated based on a typical patient profile (female, aged $57 \mathrm{yrs}$, body mass index of $30 \mathrm{~kg} / \mathrm{m}^{2}$, baseline CDAI of 20,1 prior biologic, and no comorbidities other than RA). Secondary outcomes included remission and low disease activity.

Results. There were 566 ABA initiators [anti-CCP+ $(\geq 20$ units $/ \mathrm{ml}): \mathrm{n}=362$; anti-CCP- $(<20$ units/ml): $\mathrm{n}=204]$ and 1715 TNFi initiators (anti-CCP+: $\mathrm{n}=1113$; anti-CCP-: $\mathrm{n}=602$ ). Differences between treatment groups included baseline disease duration, CDAI, and prior biologic use. At 6 months, anti-CCP+ ABA initiators were associated with significantly greater CDAI response versus anti-CCP- ABA initiators; no significant difference was observed for TNFi initiators. When considering a typical RA patient profile, $\mathrm{CDAI}$ response was greater in anti- $\mathrm{CCP}+$ versus anti-CCP- $\mathrm{ABA}$ initiators; anti-CCP+ versus anti-CCP- TNFi initiators were similar. Secondary outcome responses were also greater in anti-CCP+ versus anti-CCP- ABA initiators; TNFi initiators did not differ by anti-CCP status.

Conclusion. In a US-based clinical practice setting, anti-CCP status was associated with a differential treatment response to ABA, but not TNFi. (First Release November 1 2017; J Rheumatol 2018; 45:32-9; doi:10.3899/jrheum.170007)
\end{abstract}

Key Indexing Terms:

DISEASE-MODIFYING ANTIRHEUMATIC DRUGS ANTICYCLIC CITRULLINATED ANTIBODIES
RHEUMATOID ARTHRITIS ANTI-TUMOR NECROSIS FACTOR
The goal of rheumatoid arthritis (RA) therapy is to reduce disease activity and improve functional outcomes ${ }^{1}$. While numerous treatment options exist, identifying how to select the most effective treatment for each individual patient is not fully defined. The goal of personalized medicine is to tailor treatment to the individual characteristics of each patient. In patients with RA, it would be beneficial to identify a reliable biomarker that is associated with treatment outcomes. Anticitrullinated protein antibodies (ACPA) are sensitive and highly specific biomarkers for $\mathrm{RA}^{2}$ and are routinely assessed in clinical practice because they are part of the 2010 American College of Rheumatology (ACR)/European
From the University of Massachusetts Medical School, Worcester; Corrona LLC, Southborough, Massachusetts; Bristol-Myers Squibb, Princeton, New Jersey; Albany Medical College and The Center for Rheumatology, Albany, New York.

Sponsored by Corrona LLC. Dr. Harrold is an employee of the University of Massachusetts Medical School and Corrona LLC (with stock options). Dr. Litman, W. Hua, and S. Rebello are employees of Corrona LLC. Dr. Connolly, Dr. Kelly, E. Alemao, and Dr. Rosenblatt have stock options/bond holdings in and are employees of Bristol-Myers Squibb. Dr. Kremer has stock options/bond holdings in and is an employee of Corrona $L L C$.

L.R. Harrold, MD, MPH, University of Massachusetts Medical School, and Corrona LLC; H.J. Litman, PhD, Corrona LLC; S.E. Connolly, PhD,
Bristol-Myers Squibb; S. Kelly, MD, Bristol-Myers Squibb; W. Hua, MS, Corrona LLC; E. Alemao, MS, RPh, Bristol-Myers Squibb; L. Rosenblatt, MD, MPH, Bristol-Myers Squibb; S. Rebello, MPH, Corrona LLC; J.M. Kremer, MD, Corrona LLC, and Albany Medical College and The Center for Rheumatology.

Address correspondence to Dr. L.R. Harrold, Department of Medicine and Orthopedics, University of Massachusetts Medical School, 55 Lake Ave. North, Worcester, Massachusetts 01655, USA. E-mail: Leslie.Harrold@umassmed.edu

Full Release Article. For details see Reprints and Permissions at jrheum.org

Accepted for publication July 28, 2017. 
League Against Rheumatism diagnostic criteria ${ }^{3,4}$. Patients who are ACPA-positive tend to develop more severe, erosive disease than ACPA-negative patients ${ }^{5,6}$.

Response to RA therapy may vary based on ACPA status ${ }^{6}$. In the Abatacept versus adaliMumab comParison in bioLogic-naïvE rheumatoid arthritis subjects with background methotrexate (AMPLE; NCT00929864) trial, abatacept (ABA) was more effective in change in the 28-joint Disease Activity Score (C-reactive protein; DAS28-CRP) and the Health Assessment Questionnaire-Disability Index (HAQ-DI) in patients who were positive for anticyclic citrullinated peptide antibodies (anti-CCP, an ACPA surrogate) than in those who were negative for anti-CCP at baseline ${ }^{7}$. In addition, greater responses in DAS28 and HAQ-DI were observed in patients with higher baseline anti-CCP levels treated with $\mathrm{ABA}^{7}$. Data from the Assessing Very Early Rheumatoid arthritis Treatment (AVERT) trial also demonstrated greater clinical efficacy in ABA initiators who were anti-CCP+ for the immunoglobulin $\mathrm{M}$ isotype at baseline ${ }^{8}$.

Additional real-world data are required to analyze the association of ACPA status and the treatment effect of biological disease-modifying antirheumatic drugs (bDMARD) because patients in clinical trials are a select population and may not reflect the heterogeneous population seen in typical practice settings. To the best of our knowledge, there are no prior observational studies in the United States analyzing this relationship in patients with RA. Our study was performed to assess whether baseline anti-CCP status is predictive of response in patients initiating $\mathrm{ABA}$ or a tumor necrosis factor- $\alpha$ inhibitor (TNFi) in a US national observational cohort.

\section{MATERIALS AND METHODS}

Patient population. The Corrona RA registry is an independent, prospective, national, observational cohort in which treatment and outcomes data for patients with RA are collected and analyzed. Patients are recruited from 168 private and academic practice sites across 40 states in the United States, with 654 participating rheumatologists. As of March 31, 2016, the Corrona database included information on about 42,621 patients with RA. Data on 321,001 patient visits and 141,984 patient-years of followup observation time have been collected. The Corrona registry was established in accordance with the Declaration of Helsinki. All participating investigators were required to obtain full board approval for conducting noninterventional research involving human subjects. Sponsor approval and continuing review was obtained through a central Institutional Review Board (IRB; New England Independent Review Board, NEIRB No. 02-021). For academic investigative sites that did not receive a waiver to use the central IRB, full board approval was obtained from the respective governing IRB and documentation of approval was submitted to Corrona prior to initiating any study procedures. All registry patients were required to provide written informed consent and authorization prior to participating. The cohort of patients who met study inclusion criteria for these analyses represent 94 Corrona sites.

Study population. This analysis included adult patients with RA who initiated ABA or a TNFi (adalimumab, certolizumab pegol, etanercept, golimumab, or infliximab) during followup in the Corrona RA registry between June 2004 and January 2015. Eligible patients had to have the following: a followup visit 6 months $( \pm 3$ mos $)$ after the initiation of ABA or TNFi treatment (visit window: 3-9 mos using visit closest to 6 mos if there were $>1$ visit); Clinical Disease Activity Index (CDAI) measured at or prior to the initiation of treatment and within the appropriate timeframe prior to the visit (for initiations between visits, CDAI measured within 4 months prior to initiation was used). For the followup CDAI, it was measured at the 6-month visit or at the time of switch if patients switched medications prior to the 6-month visit. The anti-CCP status was measured at or prior to initiation.

Measures and data collection. Data were collected from both patients and their treating rheumatologists using standard clinical research forms at the time of the clinical encounter, which gather information on disease severity and activity [including serologic markers (anti-CCP) and components of ACR response criteria], comorbidities, use of medications including steroids, conventional non-TNF biologics or targeted synthetic DMARD and bDMARD, and adverse events. As a strictly observational registry that reflects typical clinical practice, Corrona does not mandate that laboratory studies including serologic markers and acute-phase response reactants be collected. Among the data elements collected in the registry relevant to this study are modified (m)ACR20, mACR50, and mACR70 (mACR based on 2 out of 4 measures; does not include erythrocyte sedimentation rate or CRP) and modified (m)HAQ assessing physical function ${ }^{9}$. Data on demographics, insurance status, comorbid conditions, RA disease characteristics, and RA medications were available for $>98 \%$ of patients. The scope of the data collected and comparison with other registries have been described previously ${ }^{10}$.

Study outcomes. Patient demographics, baseline characteristics, and clinical outcomes by anti-CCP status (anti-CCP+ and anti-CCP-) for ABA and TNFi initiators were evaluated. The primary outcome was change in CDAI score from initiation (baseline) to 6 months. Secondary outcomes included achievement of CDAI remission $[\mathrm{CDAI} \leq 2.8$; among those with low, moderate, or high disease activity $(\mathrm{CDAI}>2.8)$ at baseline] and low disease activity (LDA; CDAI $\leq 10)$ at 6 months [among those with moderate or high disease activity (CDAI $>10)$ at baseline; change from initiation to 6 months in mHAQ score; mACR20, mACR50, and mACR70].

Statistical analysis. Anti-CCP status was defined as either anti-CCP+ $(\geq 20$ units/ml) or anti-CCP- $(<20$ units $/ \mathrm{ml})$ based on the cutoffs provided by the laboratories working with the participating sites. Baseline comparisons based on anti-CCP status were conducted in both treatment groups using the Wilcoxon rank-sum test or t tests for continuous variables, and chi-square tests for categorical variables. Unadjusted response rates by anti-CCP group were estimated at 6 months after initiation of bDMARD therapy. For binary outcomes, if a patient switched to any biological agent prior to 6 months, the patient was assumed to be a nonresponder. For continuous outcomes, if a patient switched prior to 6 months, change at the time of switch was used. Adjusted response rates by anti-CCP group were determined using multivariable linear and logistic regression models that controlled for factors differing at initiation, as well as based on a priori knowledge of clinical factors thought to possibly differ by anti-CCP group [age, sex, body mass index (BMI), baseline CDAI score (baseline mHAQ score in mHAQ model), comorbidity index, and number of prior bDMARD]. A modified Charlson comorbidity index was controlled for rather than each individual measure of comorbid conditions (e.g., history of hypertension, diabetes, malignancy, or cardiovascular disease). To assess differences in binary outcomes, multivariable logistic regression models used OR and their corresponding 95\% CI. To assess differences in continuous outcomes, multivariable linear regression models used the mean and 95\% CI reported for each anti-CCP group. Concomitant methotrexate (MTX) and prednisone use did not meet criteria for inclusion in the multivariable models (e.g., there was no imbalance between groups). However, given their effect on treatment response, the multivariable models were re-run, with these 2 factors included in addition to the variables that met criteria as specified above; the results were unchanged (data not shown). Similarly, calendar year was analyzed in the final models. Because the results were unchanged, these are not presented.

Personal non-commercial use only. The Journal of Rheumatology Copyright $\subset$ C 2018. All rights reserved. 
The above-mentioned adjusted models did not allow for direct comparison between ABA and TNFi initiators because of baseline differences in the source populations. Therefore, we conducted additional analyses to compare those who had characteristics common in both ABA and TNFi initiators. This "typical" patient with RA was considered to be female, aged 57 years, with a BMI of $30 \mathrm{~kg} / \mathrm{m}^{2}$, baseline CDAI of 20 , who had received 1 prior biological agent, and had no comorbidities aside from RA according to the modified Charlson Comorbidity Index. We evaluated treatment response for this prototype using separate linear regression models for ABA and TNFi initiators. Patients with missing values for covariates included in the multivariable linear and logistic regression models were excluded from the models (on average we lost $<1 \%$ of the sample in the adjusted analyses).

\section{RESULTS}

Patient disposition and baseline characteristics. Baseline demographics and clinical characteristics by anti-CCP status are shown in Table 1. Of the 2835 patients who initiated ABA, 2525 patients had a CDAI measurement at baseline, 2124 had a 6-month visit within the \pm 3 -month visit window, 2101 had CDAI at both baseline and at 6-month followup, and 566 patients also had anti-CCP measured at or prior to initiation: $204(36.0 \%)$ were anti-CCP- and 362 (64.0\%) were anti-CCP+ (Appendix 1). Patients who had anti-CCP testing were similar to those without anti-CCP testing in terms of female sex ( $81 \%$ vs $82 \%$ ), median age (58 vs 60 yrs), and median CDAI (21). They were slightly more likely to be biologic-experienced ( $87 \%$ vs $84 \%$ ) and had fewer median years of disease duration ( $7 \mathrm{vs} 10)$. Among those with $\mathrm{CCP}$ testing, there were no differences by anti-CCP status for age (median $58 \mathrm{yrs}$ ), disease duration (median $7 \mathrm{yrs}$ ), CDAI (median 22 vs 21 ), or prior TNFi use $(0,1$, or $\geq 2$ prior agents; Table 1).

Of the 8343 patients who initiated TNFi, 7481 patients had a CDAI measurement at baseline, 6139 had a 6-month visit within the \pm 3 -month visit window, 6021 patients had CDAI at baseline and a 6-month followup, and 1715 had anti-CCP results at or prior to initiation: $602(35.1 \%)$ were anti-CCP- and 1113 (64.9\%) were anti-CCP+ (Appendix 1). Patients who had anti-CCP testing were similar to those without anti-CCP testing in terms of female sex (77\% vs $78 \%$ ), median age (56 vs $57 \mathrm{yrs}$ ), and median CDAI (19); however, they had fewer median years of disease duration (4 vs 6) and were less likely to be biologic-experienced (33\% vs $40 \%$ ). Among those with anti-CCP testing, there were no differences by CCP status for age (median 55-56 yrs), prior TNFi use, or median baseline CDAI; however, median disease duration was greater in anti-CCP+ versus anti-CCPpatients (median 4 vs 3 yrs, $p=0.021$; Table 1 ). In general, the ABA and TNFi patient populations were different in terms of disease duration, CDAI, and prior use of biological agents (Table 1).

Mean change from baseline in disease activity and disability. For patients who initiated ABA, anti-CCP+ status was associated with a significantly greater response at 6 months compared with anti-CCP- status on all outcomes measures. For the primary outcome, anti-CCP+ ABA initiators had a mean $(95 \% \mathrm{CI})$ change in CDAI of -8.5 ( -9.9 to -7.0$)$ versus $-4.3(-6.2$ to $-2.3, \mathrm{p}=0.001)$ for those with an anti-CCPstatus in unadjusted analyses (Figure 1). After adjusting for baseline covariates, significant differences in CDAI change from baseline between the anti-CCP+ and the anti-CCPgroups remained $[-8.5(-9.7$ to -7.3$)$ vs -4.0 ( -5.6 to -2.4$)$, $\mathrm{p}<0.001$; Figure 1]. Conversely, there were no significant differences in mean $(95 \% \mathrm{CI})$ change in CDAI between the anti-CCP+ TNFi initiators and the anti-CCP-TNFi initiators in unadjusted $[-7.3(-8.1$ to -6.4$)$ vs $-6.8(-7.9$ to -5.7$)$, $\mathrm{p}=0.52]$ and adjusted analyses $[-7.4(-8.1$ to -6.8$)$ vs -6.4 $(-7.3$ to -5.6$), p=0.072$; Figure 2]. Significant differences in mean $(95 \% \mathrm{CI})$ change in mHAQ [ $-0.12(-0.16$ to -0.07$)$ vs $0.00(-0.06$ to 0.06$), \mathrm{p}=0.001]$ were observed for anti-CCP+ $\mathrm{ABA}$ initiators versus anti-CCP- $\mathrm{ABA}$ initiators in unadjusted analyses. After adjusting for baseline covariates, significant differences in mHAQ remained. There were no significant differences in mHAQ for TNFi initiators by anti-CCP status in unadjusted $[-0.08(-0.11$ to -0.04$)$ vs $-0.08(-0.10$ to -0.05$), \mathrm{p}=0.99]$ or adjusted analyses [ -0.07 $(-0.10$ to -0.04$)$ vs $-0.08(-0.10$ to -0.06$), \mathrm{p}=0.54]$.

Mean change from baseline in CDAI for $A B A$ and TNF $i$ initiators with the same "typical" RA patient profile. A typical patient was considered to provide information about the same patient population because the 2 groups differed according to baseline CDAI, disease duration, number of prior biological agents, and other baseline covariates. When considering treatment response based on a typical RA patient profile, the mean (SE) change in CDAI from baseline at 6 months in the anti-CCP+ patients was numerically greater than that in anti-CCP- patients among ABA initiators [-8.8 (0.8) vs -4.4 (0.9); Figure 3]. When considering the same typical RA patient profile among TNFi initiators, the mean change in CDAI of $-4.6(0.5)$ in anti-CCP- patients was similar to the anti-CCP- patients who were $\mathrm{ABA}$ initiators, but the mean change in $\mathrm{CDAI}$ from baseline was $-5.6(0.4)$ for anti-CCP+ patients who were TNFi initiators (Figure 3).

Remission and LDA. For ABA initiators, anti-CCP+ status was significantly associated with greater remission responses $[\mathrm{CDAI} \leq 2.8$ among those with low, moderate, or high disease activity who initiated treatment $(\mathrm{n}=544)]$ compared with anti-CCP- status $(15.2 \%$ vs $4.6 \%, \mathrm{p}<0.001)$. A greater proportion of anti-CCP+ ABA initiators achieved LDA $[C D A I \leq 10$ among those with moderate or high disease activity who initiated treatment $(n=460)]$ compared with those with an anti-CCP- status initiating ABA (41.4\% vs $24.1 \%, \mathrm{p}<0.001)$. After adjusting for baseline covariates, the odds of achieving remission and LDA were significantly higher for those with anti-CCP+ status versus anti-CCPstatus (Figure 4). The proportion of patients attaining remission $(14.9 \%$ vs $14.4 \%, \mathrm{p}=0.76)$ and the odds of achieving remission after adjusting for baseline covariates did not differ by anti-CCP status in TNFi initiators (Figure

Personal non-commercial use only. The Journal of Rheumatology Copyright (C) 2018. All rights reserved. 
Table 1. Baseline patient demographics and clinical characteristics by anti-CCP status ${ }^{\dagger}$ among all ABA and TNFi initiators. Values are $\mathrm{n}(\%)$ unless otherwise specified.

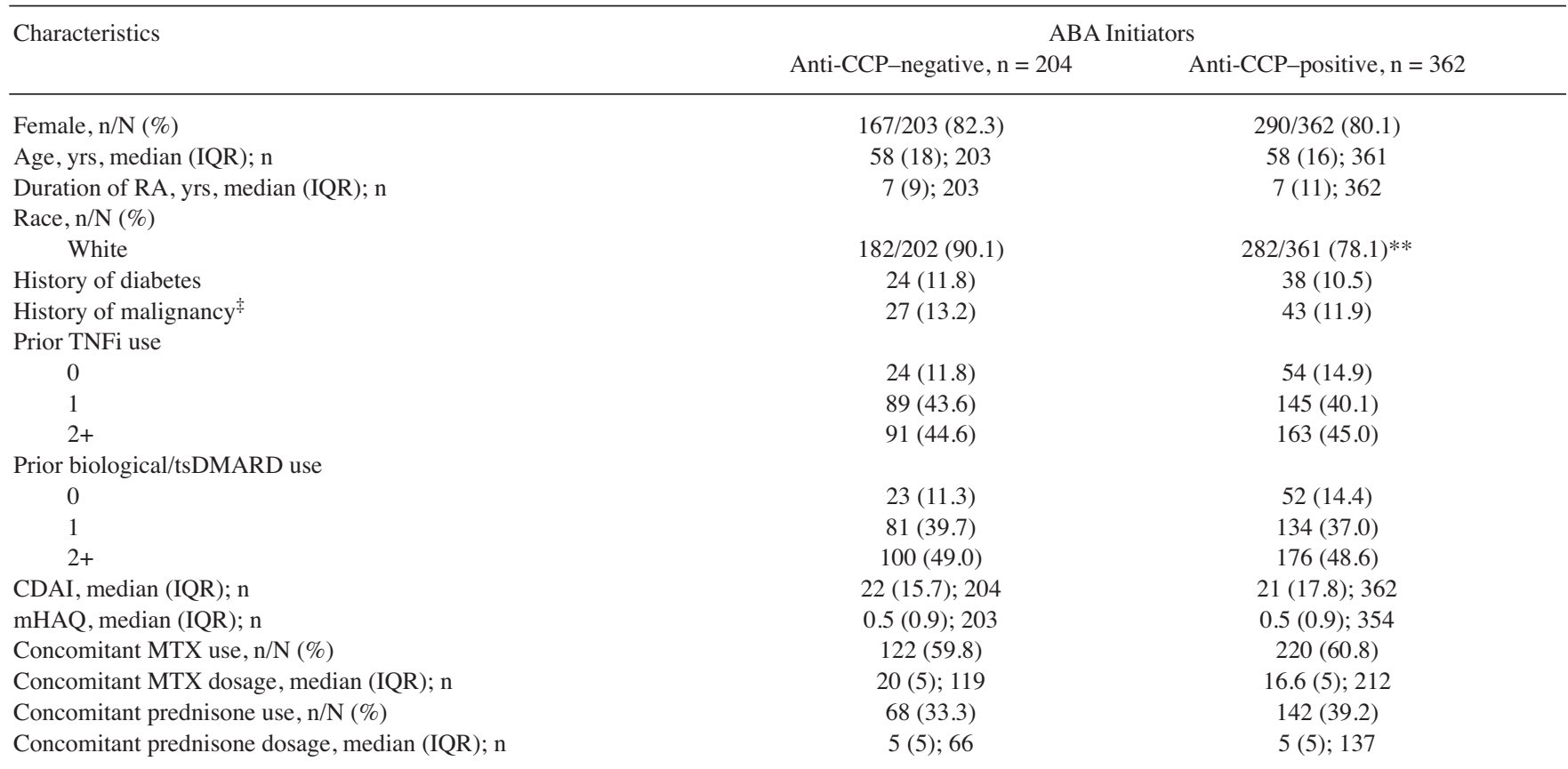

$$
\text { Anti-CCP-negative, } \mathrm{n}=602 \quad \text { Anti-CCP-positive, } \mathrm{n}=1113
$$

\begin{tabular}{|c|c|c|}
\hline Female, $\mathrm{n} / \mathrm{N}(\%)$ & $474 / 601(78.9)$ & $843 / 1113(75.7)$ \\
\hline Age, yrs, median (IQR); n & $55(16) ; 601$ & $56(17) ; 1111$ \\
\hline Duration of RA, yrs, median (IQR); $n$ & $3(7) ; 597$ & $4(9) ; 1108^{*}$ \\
\hline \multicolumn{3}{|l|}{ Race, $\mathrm{n} / \mathrm{N}(\%)$} \\
\hline White & $507 / 598(84.8)$ & $903 / 1113(81.1)^{* *}$ \\
\hline History of diabetes & $42(7.0)$ & $89(8.0)$ \\
\hline History of malignancy & $53(8.8)$ & $63(5.7)^{*}$ \\
\hline \multicolumn{3}{|l|}{ Prior TNFi use } \\
\hline 0 & $406(67.4)$ & $795(71.4)$ \\
\hline 1 & $144(23.9)$ & $242(21.7)$ \\
\hline $2+$ & $52(8.6)$ & $76(6.8)$ \\
\hline \multicolumn{3}{|l|}{ Prior biological/tsDMARD use } \\
\hline 0 & $384(63.8)$ & $762(68.5)$ \\
\hline 1 & $139(23.1)$ & $247(22.2)$ \\
\hline $2+$ & $79(13.1)$ & $104(9.3)$ \\
\hline CDAI, median (IQR); $\mathrm{n}$ & $19(20.6) ; 602$ & $18(18.0) ; 1113$ \\
\hline mHAQ, median (IQR); $\mathrm{n}$ & $0.4(0.8) ; 602$ & $0.4(0.8) ; 1102$ \\
\hline Concomitant MTX use, n/N (\%) & $391(65.0)$ & $746(67.0)$ \\
\hline Concomitant MTX dosage, median (IQR); n & $20(5) ; 376$ & $20(5) ; 706$ \\
\hline Concomitant prednisone use, $\mathrm{n} / \mathrm{N}(\%)$ & $150(24.9)$ & $318(28.6)$ \\
\hline Concomitant prednisone dosage, median (IQR); $\mathrm{n}$ & $5(3.3) ; 147$ & $5(5) ; 308$ \\
\hline
\end{tabular}

${ }^{\dagger}$ Anti-CCP status was defined as either anti-CCP-positive $\left(\geq 20\right.$ units/ml) or anti-CCP-negative $\left(<20\right.$ units/ml). ${ }^{\ddagger}$ History of malignancy includes history of lung cancer, breast cancer, skin cancer, lymphoma, or other cancer. *p value $\leq 0.05$. **p value $\leq 0.01$. ABA: abatacept; TNFi: tumor necrosis factor- $\alpha$ inhibitor; anti-CCP: anticyclic citrullinated peptide antibodies; IQR: interquartile range; RA: rheumatoid arthritis; tsDMARD: targeted synthetic disease-modifying antirheumatic drug; CDAI: Clinical Disease Activity Index; mHAQ: modified Health Assessment Questionnaire; MTX: methotrexate.

5). However, the proportion of TNFi initiators achieving LDA was higher in the anti-CCP+ group versus the anti-CCP-group in the unadjusted model $(40.6 \%$ vs $34.1 \%$, $\mathrm{p}=0.023)$; this effect was no longer significant after adjusting for baseline covariates (Figure 5). $m A C R$ response rates. Anti- $\mathrm{CCP}+\mathrm{ABA}$ initiators had significantly greater mACR responses versus those with an anti-CCP- status (mACR20: $34.7 \%$ vs $22.0 \%$; mACR50: $23.3 \%$ vs $9.0 \%$; mACR70: $12.2 \%$ vs $4.0 \%, \mathrm{p}<0.01$ for all). After adjusting for baseline covariates, the differences 


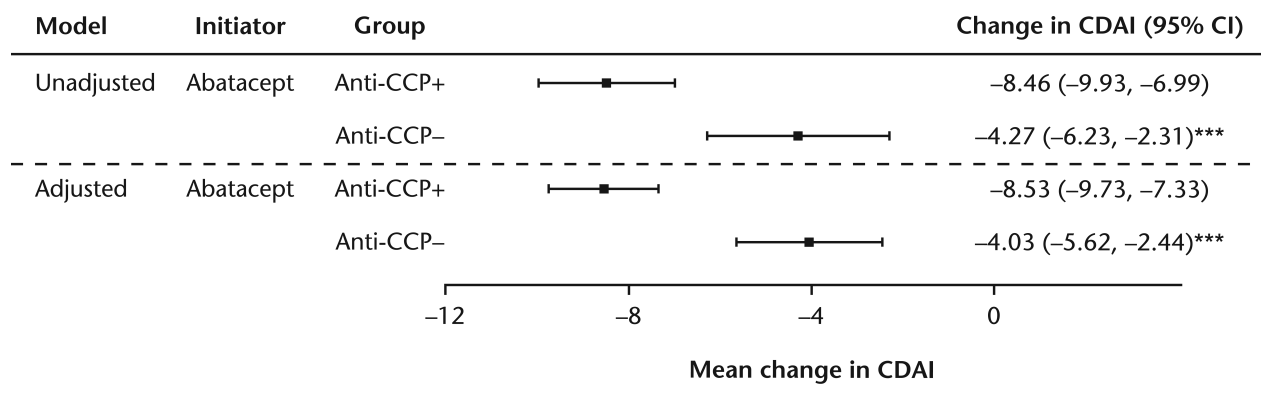

Figure 1. Mean change from baseline in disease and disability outcomes at 6 months for abatacept initiators by anti-CCP status. Model adjusted by baseline age, sex, body mass index, CDAI score, comorbidity index, and number of prior biological DMARD. $* * * \mathrm{p} \leq 0.001$. Anti-CCP status was defined as either anti-CCP-positive ( $\geq 20$ units $/ \mathrm{ml}$ ) or anti-CCP-negative $(<20$ units $/ \mathrm{ml})$. Patient numbers: unadjusted anti-CCP+, $\mathrm{n}=362$; unadjusted anti-CCP-, $\mathrm{n}=204$; adjusted anti-CCP+, $\mathrm{n}=361$; adjusted anti-CCP-, $\mathrm{n}=203$. Anti-CCP: anticyclic citrullinated peptide antibodies; CDAI: Clinical Disease Activity Index; DMARD: disease-modifying antirheumatic drug.

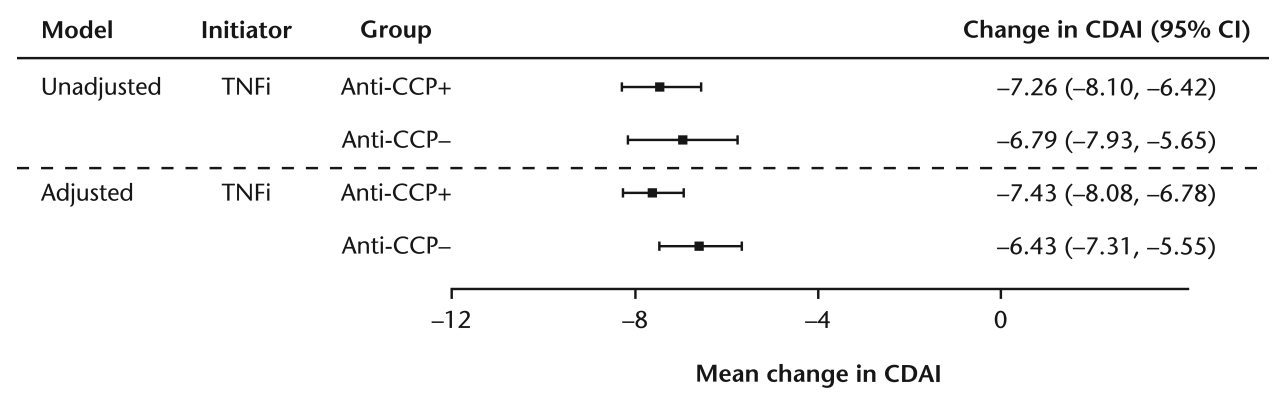

Figure 2. Mean change from baseline in disease and disability outcomes at 6 months for TNFi initiators by anti-CCP status. Model adjusted by baseline age, sex, body mass index, CDAI score, comorbidity index, and number of prior biological DMARD. All $\mathrm{p}$ values $>0.05$. Anti-CCP status was defined as either anti-CCPpositive $(\geq 20$ units $/ \mathrm{ml})$ or anti-CCP-negative $(<20$ units $/ \mathrm{ml})$. Patient numbers: unadjusted anti-CCP+, $\mathrm{n}=$ 1113; unadjusted anti-CCP-, $n=602$; adjusted anti-CCP+, $n=1110$; adjusted anti-CCP-, $n=600$. Anti-CCP: anticyclic citrullinated peptide antibodies; CDAI: Clinical Disease Activity Index; DMARD: disease-modifying antirheumatic drug; TNFi: tumor necrosis factor- $\alpha$ inhibitor.

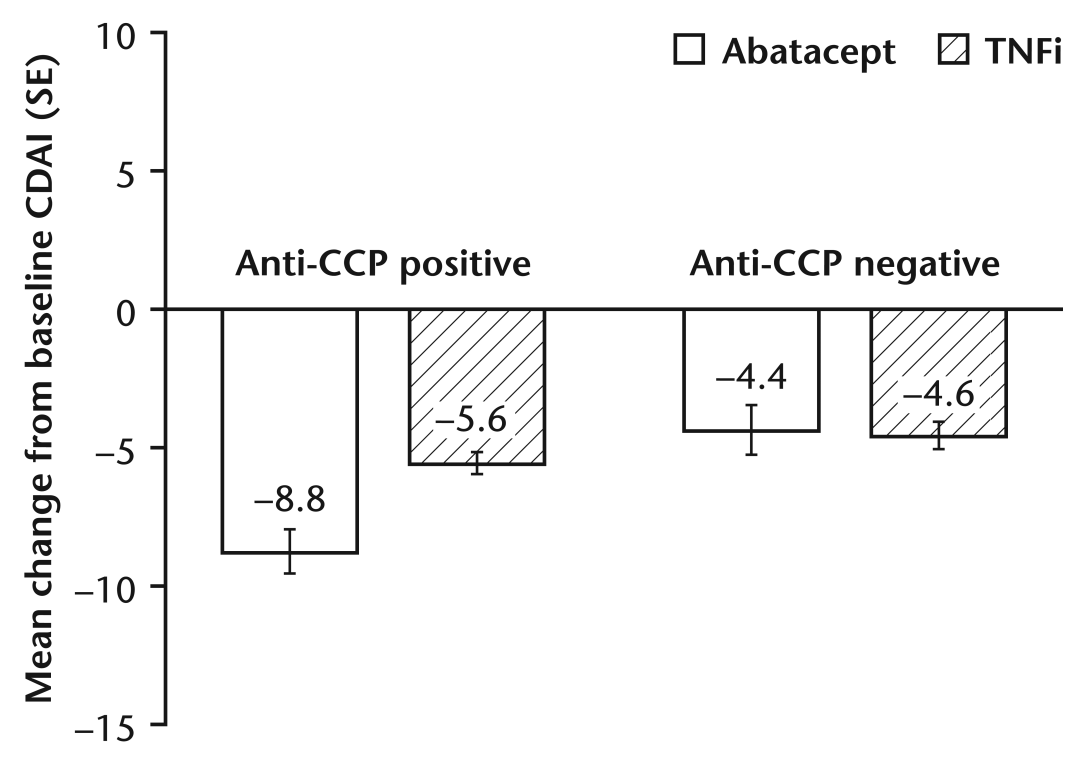

Figure 3. Mean change from baseline CDAI in abatacept and TNFi initiators with a typical patient profile. The typical patient was defined as a female, aged 57 years, with a body mass index of $30 \mathrm{~kg} / \mathrm{m}^{2}$, and a baseline CDAI of 20 , who had received 1 prior biological agent and had no comorbidities other than rheumatoid arthritis on the modified Charlson comorbidity index. Anti-CCP status was defined as either anti-CCP-positive ( $\geq 20$ units/ml) or anti-CCP-negative (< 20 units $/ \mathrm{ml}$ ). Patient numbers: abatacept anti-CCP+, $\mathrm{n}=361$; abatacept anti-CCP-, $\mathrm{n}=203$; TNF anti-CCP+, $\mathrm{n}=1110$; TNF anti-CCP-, $n=600$. Anti-CCP: anticyclic citrullinated peptide antibodies; CDAI: Clinical Disease Activity Index; TNFi: tumor necrosis factor- $\alpha$ inhibitor. 


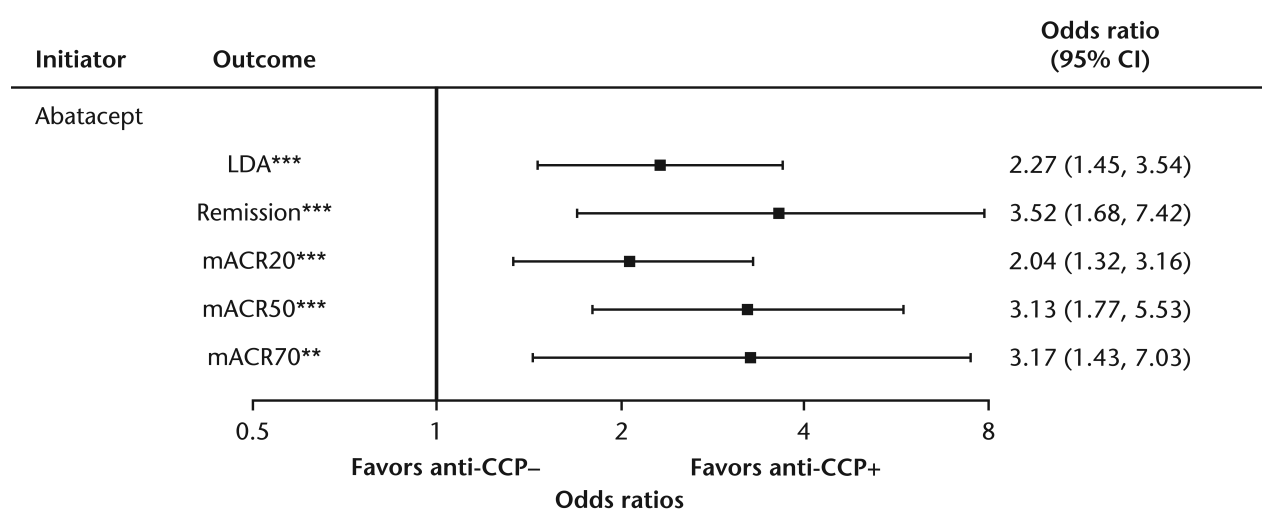

Figure 4. Association between anti-CCP antibody status and response to abatacept (multivariable models). Model adjusted by baseline age, sex, body mass index, CDAI score, comorbidity index, and number of prior biological DMARD. $* * \mathrm{p}<0.01$. ***p $\leq 0.001$. Anti-CCP status was defined as either anti-CCP-positive ( $\geq 20 \mathrm{units} / \mathrm{ml}$ ) or anti-CCP-negative $(<20$ units $/ \mathrm{ml})$. LDA was defined as CDAI $\leq 10$ among those with moderate or high disease activity $(\mathrm{CDAI}>10)$ who initiated treatment $(n=460)$. Remission was defined as CDAI $\leq 2.8$ among those with low, moderate, or high disease activity $(\mathrm{CDAI}>2.8)$ who initiated treatment $(\mathrm{n}=544)$. Modified ACR was based on 2 out of 4 measures and does not include erythrocyte sedimentation rate or $\mathrm{C}$-reactive protein. Patient numbers: LDA, anti-CCP-, $\mathrm{n}=170$, anti-CCP+, $\mathrm{n}=290$; remission, anti-CCP-, $\mathrm{n}=195$, anti-CCP+, $\mathrm{n}=349$; mACR20, anti-CCP-, $\mathrm{n}=200$, anti-CCP+, $\mathrm{n}=352 ; \mathrm{mACR} 50$, anti-CCP-, $\mathrm{n}=200$, anti-CCP+, $\mathrm{n}=352 ; \mathrm{mACR} 70$, anti-CCP,$n=200$, anti-CCP+, $n=352$. Anti-CCP: anticyclic citrullinated peptide antibodies; CDAI: Clinical Disease Activity Index; DMARD: disease-modifying antirheumatic drug; LDA: low disease activity; mACR: modified American College of Rheumatology.

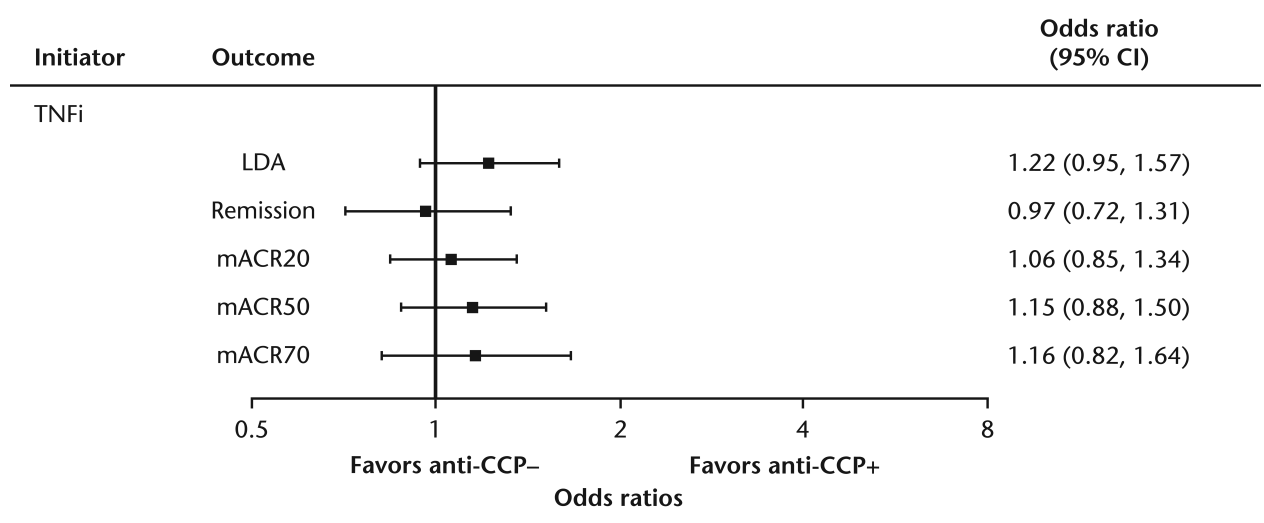

Figure 5. Association between anti-CCP antibody status and response to remission, LDA and mACR by anti-CCP status and TNFi initiators (multivariable models). Model adjusted by baseline age, sex, body mass index, CDAI score, comorbidity index, and number of prior biological DMARD. All p values $>0.10$. Anti-CCP status was defined as either anti-CCP-positive $(\geq 20 \mathrm{units} / \mathrm{ml})$ or anti-CCP-negative $(<20 \mathrm{units} / \mathrm{ml})$. LDA was defined as $\mathrm{CDAI} \leq 10$ among those with moderate or high disease activity $(\mathrm{CDAI}>10)$ who initiated treatment $(\mathrm{n}=1278)$. Remission was defined as CDAI $\leq 2.8$ among those with low, moderate, or high disease activity $(\mathrm{CDAI}>2.8)$ who initiated treatment $(n=1601)$. mACR was based on 2 out of 4 measures and does not include erythrocyte sedimentation rate or $\mathrm{C}$-reactive protein. Patient numbers: $\mathrm{LDA}$, anti-CCP-, $\mathrm{n}=451$, anti-CCP, $\mathrm{n}=827$; remission, anti-CCP-, $\mathrm{n}=577$, anti-CCP+, $\mathrm{n}=1024$; mACR20, anti-CCP- $\mathrm{n}=598$, anti-CCP+, $\mathrm{n}=1087$; mACR50, anti-CCP-, $\mathrm{n}=598$, anti-CCP+, $\mathrm{n}=1087$; mACR70: anti-CCP-, $\mathrm{n}=598$, anti-CCP+, $\mathrm{n}=1087$. Anti-CCP: anticyclic citrullinated peptide antibodies; LDA: low disease activity; mACR: modified American College of Rheumatology; TNFi: tumor necrosis factor- $\alpha$ inhibitor; CDAI: Clinical Disease Activity Index; DMARD: disease-modifying antirheumatic drug.

between the anti-CCP+ and the anti-CCP- groups remained statistically significant, and the odds of achieving mACR response rates were significantly higher for those with an anti-CCP+ status versus those with an anti-CCP- status (Figure 4).
For TNFi initiators, there were no statistically significant differences in mACR responses by anti-CCP status in unadjusted (mACR20: $31.6 \%$ vs $31.1 \%$; mACR50: $19.9 \%$ vs $17.7 \%$; mACR70: $10.6 \%$ vs $8.9 \%)$ and adjusted analyses $(\mathrm{p}>0.10$; Figure 5).

Personal non-commercial use only. The Journal of Rheumatology Copyright @ 2018 . All rights reserved. 


\section{DISCUSSION}

These real-world data from US clinical practices demonstrate that baseline anti-CCP status is associated with clinical response to $\mathrm{ABA}$, with the better clinical response observed in anti-CCP+ patients. In contrast, no such relationship was evident between anti-CCP status and response to a TNFi. When considering a typical RA patient profile, anti-CCP+ status was associated with greater improvements in response among ABA initiators compared with anti-CCP-patients. In contrast, among TNFi initiators with the same typical RA profile, responses were similar among anti-CCP+ and anti-CCP- patients; the magnitude of change in CDAI in anti-CCP- patients was similar to that in the anti-CCPpatients initiating $\mathrm{ABA}$. The underlying reason for the observed differences in response for $\mathrm{ABA}$ and TNFi is unknown, but may be related to differences in the mechanism of action between treatments. The association between seropositivity and ABA response could be due to the upstream mechanism of action of ABA, by which costimulation blockade inhibits Th cell activation and subsequently modulates B cells and autoantibody production ${ }^{11,12}$. Anti-CCP is associated with erosive disease ${ }^{5}$, and therefore it is important to have a reliable biomarker to help identify patients who will benefit from treatment response.

Other studies have similarly shown antibody seropositivity to be related to response to ABA. In the ACTION study, anti-CCP-positive and/or RF-positive status was associated with greater efficacy of ABA than seronegative status ${ }^{13}$. Of note, we were unable to evaluate the independent effect of RF because we had too few patients who were RF-positive and anti-CCP-negative. Additionally, these data are generally consistent with ACPA results from the AVERT and AMPLE trials. ABA in combination with MTX had a greater clinical efficacy in patients who exhibited a greater level of serocomplexity at baseline (either higher titers or a greater number of isotypes $)^{8,14}$. However, data from the AMPLE trial show baseline anti-CCP positivity was also associated with a better response for adalimumab, whereas in the current study generally no consistent differences were seen by anti-CCP status for $\mathrm{TNFi}^{7}$. These differences may be due to different patient populations between the studies because overall, the patients in AMPLE had more severe RA than did patients in the Corrona database, and were biologic-naive with an inadequate response to MTX.

There have been conflicting results, with some studies showing anti-CCP associated with a reduced response to TNFi, and other studies demonstrating improved results 6,15 . A systematic literature review of 14 studies (13 prospective clinical trials and 1 retrospective study) investigated whether the status of anti-CCP in patients with RA has a predictive value for clinical response to TNFi therapy. Consistent with our present study, the metaanalysis demonstrated that anti-CCP was not found to be associated with a response to $\mathrm{TNFi}^{6}$.
The strengths of our study are that these data are from a large, national, observational cohort of patients with RA and includes both patient-reported and clinical measures such as serological status. Limitations of our study should be noted. This is a real-world patient population cared for by rheumatologists across the United States; thus, there is no centralized laboratory evaluating serological status. This potential variability in serological testing was minimized in our study by categorizing patients according to anti-CCP status (e.g., positive vs negative) rather than analyzing by anti-CCP titers. The patient population primarily reflects white females; therefore, future analyses should be conducted in other racial and ethnic groups. In addition, this is a registry cohort and the results depend on the RA characteristics of the patients who entered the cohort and for whom laboratory results were available. However, it is unlikely that there is a systematic bias between patients enrolled with laboratory results and without, and no reason to believe that if such a bias existed that it would differ according to treatment. Nonetheless, this is a routine clinical care setting and demonstrates real-world outcomes of RA more precisely than would an interventional clinical trial (e.g., disease activity is not typically as high and there is no exclusion of comorbidities).

Our results show that, in routine clinical practice, anti-CCP positivity was associated with treatment response to ABA, but not TNFi. These findings suggest that anti-CCP status may be useful for selecting appropriate therapy in patients with RA. For providers discussing with their patients the advantages and disadvantages of different therapeutic options, this laboratory information may be one of the factors to aid in decision making. The predictive value of ACPA concentration and status need to be further examined beyond our study to determine whether RA treatment could be improved by using ACPA status and other biomarkers to predict which patients will more likely respond to a particular intervention such as $\mathrm{ABA}$.

\section{ACKNOWLEDGMENT}

Professional medical writing and editorial assistance was provided by Stacey Reeber, $\mathrm{PhD}$, at Caudex and was funded by Bristol-Myers Squibb.

\section{REFERENCES}

1. Singh JA, Saag KG, Bridges SL Jr, Akl EA, Bannuru RR, Sullivan MC, et al. 2015 American College of Rheumatology guideline for the treatment of rheumatoid arthritis. Arthritis Rheumatol 2016;68:1-26.

2. Zendman AJ, van Venrooij WJ, Pruijn GJ. Use and significance of anti-CCP autoantibodies in rheumatoid arthritis. Rheumatology 2006;45:20-5.

3. van Boekel MA, Vossenaar ER, van den Hoogen FH, van Venrooij WJ. Autoantibody systems in rheumatoid arthritis: specificity, sensitivity and diagnostic value. Arthritis Res 2002;4:87-93.

4. Aletaha D, Neogi T, Silman AJ, Funovits J, Felson DT, Bingham CO 3rd, et al. 2010 Rheumatoid arthritis classification criteria: an American College of Rheumatology/European League Against Rheumatism collaborative initiative. Arthritis Rheum 2010; 62:2569-81.

Personal non-commercial use only. The Journal of Rheumatology Copyright @ 2018. All rights reserved. 
5. van der Helm-van Mil AH, Verpoort KN, Breedveld FC, Toes RE, Huizinga TW. Antibodies to citrullinated proteins and differences in clinical progression of rheumatoid arthritis. Arthritis Res Ther 2005; 7:R949-58.

6. Lv Q, Yin Y, Li X, Shan G, Wu X, Liang D, et al. The status of rheumatoid factor and anti-cyclic citrullinated peptide antibody are not associated with the effect of anti-TNF $\alpha$ agent treatment in patients with rheumatoid arthritis: a meta-analysis. PLoS One 2014;9:e89442.

7. Sokolove J, Schiff M, Fleischmann R, Weinblatt ME, Connolly SE, Johnsen A, et al. Impact of baseline anti-cyclic citrullinated peptide-2 antibody concentration on efficacy outcomes following treatment with subcutaneous abatacept or adalimumab: 2-year results from the AMPLE trial. Ann Rheum Dis 2015;74:983-4.

8. Huizinga TW, Connolly SE, Johnsen A, Zhu J, Furst DE, Bykerk VP, et al. Effect of anti-cyclic citrullinated peptide 2 immunoglobulin $\mathrm{M}$ serostatus on efficacy outcomes following treatment with abatacept plus methotrexate in the AVERT trial. Ann Rheum Dis 2015;74 Suppl 2:234.

9. Anderson J, Caplan L, Yazdany J, Robbins ML, Neogi T, Michaud $\mathrm{K}$, et al. Rheumatoid arthritis disease activity measures: American College of Rheumatology recommendations for use in clinical practice. Arthritis Care Res 2012;64:640-7.

10. Curtis JR, Jain A, Askling J, Bridges SL Jr, Carmona L, Dixon W, et al. A comparison of patient characteristics and outcomes in selected
European and U.S. rheumatoid arthritis registries. Semin Arthritis Rheum 2010;40:2-14.

11. Pieper J, Herrath J, Raghavan S, Muhammad K, Vollenhoven Rv, Malmström V. CTLA4-Ig (abatacept) therapy modulates T cell effector functions in autoantibody-positive rheumatoid arthritis patients. BMC Immunol 2013;14:34.

12. Scarsi M, Paolini L, Ricotta D, Pedrini A, Piantoni S, Caimi L, et al. Abatacept reduces levels of switched memory B cells, autoantibodies, and immunoglobulins in patients with rheumatoid arthritis. J Rheumatol 2014;41:666-72.

13. Alten R, Nüßlein HG, Mariette X, Galeazzi M, Lorenz HM, Cantagrel A, et al. Baseline autoantibodies preferentially impact abatacept efficacy in patients with rheumatoid arthritis who are biologic naive: 6-month results from a real-world, international, prospective study. RMD Open 2017;3:e000345.

14. Huizinga T, Connolly S, Furst DE, Bykerk V, Burmester G, Combe $\mathrm{BG}$, et al. The impact on anti-citrullinated protein antibody isotypes and epitope fine specificity in patients with early RA treated with abatacept and methotrexate. Arthritis Rheum 2014;66:S666.

15. Fisher BA, Plant D, Lundberg K, Charles P, Barton A, Venables PJ; Biologics in Rheumatoid Arthritis Genetics and Genomics Study Syndicate (BRAGGSS). Heterogeneity of anticitrullinated peptide antibodies and response to anti-tumor necrosis factor agents in rheumatoid arthritis. J Rheumatol 2012;39:929-32.

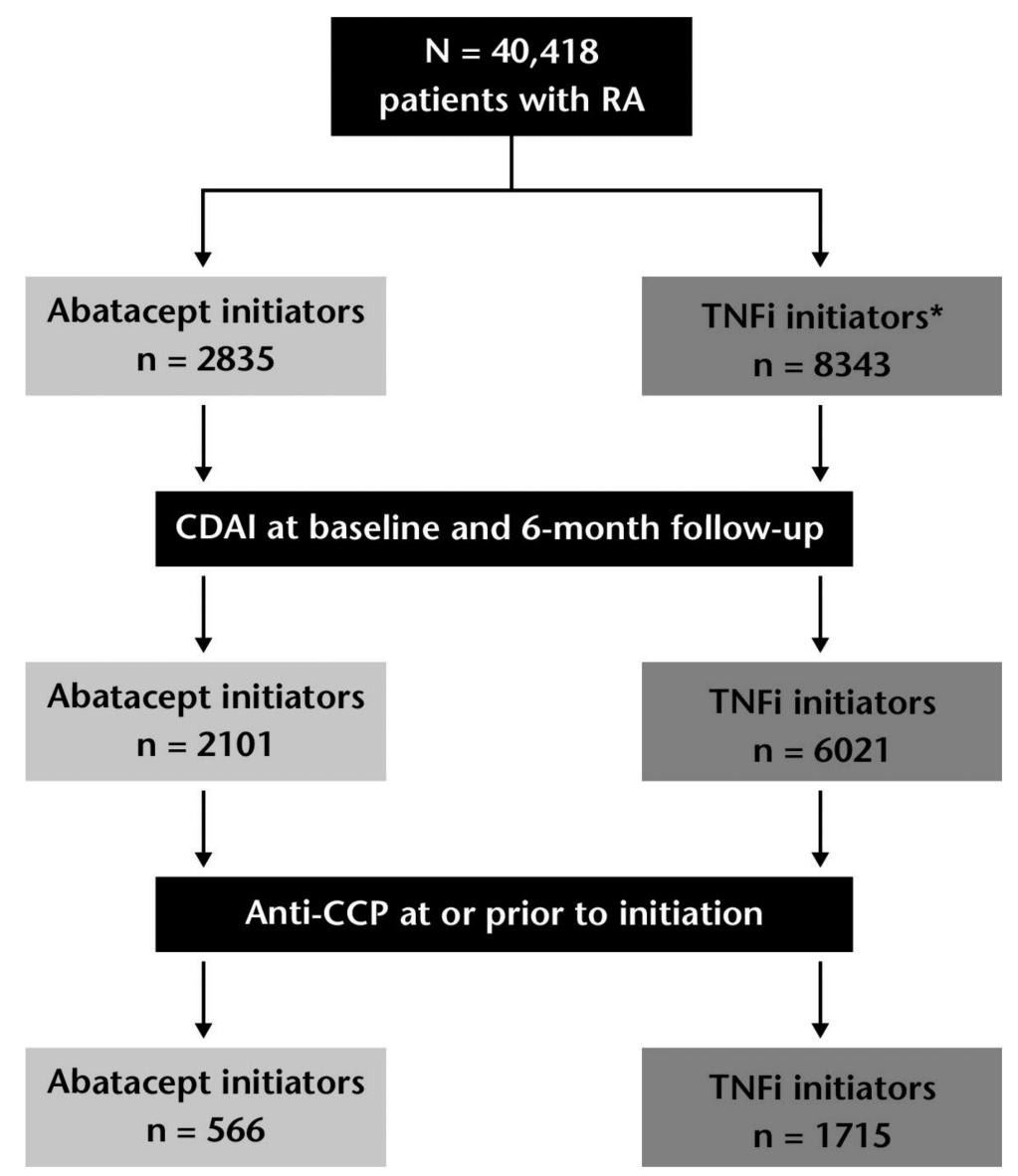

APPENDIX 1. Study population. *Adalimumab, certolizumab pegol, etanercept, golimumab, and infliximab. RA: rheumatoid arthritis; TNFi: tumor necrosis factor- $\alpha$ inhibitor; CDAI: Clinical Disease Activity Index; anti-CCP: anticyclic citrullinated peptide antibodies. 